\title{
Fatores associados à fissura em usuários de crack: revisão sistemática
}

\author{
Karine Langmantel Silveira ${ }^{1}$ \\ Michele Mandagará Oliveira² \\ Poliana Farias Alves ${ }^{3}$
}

\begin{abstract}
Objetivo: identificar publicações relacionadas à influência dos fatores associados à variação dos níveis de fissura em usuários de crack. Metodologia: revisão de literatura sistemática, em que se rastreou estudos publicados no Brasil e exterior. As bases de dados utilizadas foram Publisher Medline, Literatura Latino-Americana e do Caribe em Ciências da Saúde e Scientific Electronic Library. Resultados: foram selecionados nove artigos, após a utilização dos critérios de inclusão e exclusão. Os fatores associados observados estão relacionados ao padrão de uso do crack, relações familiares, atividade profissional, utilização de outras substâncias, estratégias de redução de danos e ansiedade. Conclusão: acredita-se que a fissura pode interferir no consumo abusivo de crack e, assim, ocasionar diversos problemas tanto de ordem social como de saúde.
\end{abstract}

Descritores: Cocaína Crack; Fissura; Problemas Sociais.

\footnotetext{
${ }^{1}$ Mestranda, Universidade Federal de Pelotas. Enfermeira. Universidade Federal de Pelotas. Bolsista CAPES.

${ }^{2}$ Doutorado, Universidade de São Paulo. Enfermeira. Universidade Federal de Pelotas, Professor Adjunto.

${ }^{3}$ Doutoranda, Universidade Federal de Pelotas. Enfermeira. Universidade Federal de Pelotas. Bolsista CAPES.
} 


\title{
Factors associated with craving in crack users: systematic review
}

Objective: to identify publications related to the influence of the factors associated with the variation in the levels of craving in crack users. Method: systematic literature review in which studies published in Brazil and abroad were screened. The databases used were Publisher Medline, Literatura Latino-Americana e do Caribe em Ciências da Saúde and Scientific Electronic Library Online. Results: nine articles were selected, after the use of the inclusion and exclusion criteria. The associated factors observed are related to the pattern of crack use, family relationships, professional activity, use of other substances, damage reduction strategies and anxiety. Conclusion: craving can interfere in crack abuse and thus cause different social and health problems.

Descriptors: Crack Cocaine; Craving; Social Problems.

\section{Los factores asociados con la ansia en los consumidores de crack:} revisión sistemática

\begin{abstract}
Objetivo: Identificar publicaciones relacionadas con la influencia de los factores asociados con la variación de los niveles de ansia en los consumidores de crack. Métodos: Una revisión sistemática de la literatura que rastreó los estudios publicados en Brasil y en el extranjero. Las bases de datos utilizadas fueron Publisher Medline, América Latina y el Caribe en Ciencias de la Salud y Scientific Electronic Library. Resultados: Se seleccionaron nueve artículos después del uso de los criterios de inclusión y exclusión. Los factores asociados observados en los estudios se relacionan a lo comportamiento de lo consumo de crack, las relaciones familiares, la ocupación, el uso de otras sustancias, estrategias de reducción de daños y la ansiedad. Conclusión: La ansia puede causar el consumo excesivo de crack y por lo tanto causar muchos problemas tanto sociales cuanto de salud.
\end{abstract}

Descriptores: Cocaína Crack; Ansia; Problemas Sociales.

\section{Introdução}

Mesmo o crack não estando entre as drogas ilícitas mais utilizadas no Brasil, a urgência pelo uso da substância e a intensidade dos efeitos da fissura colocam o uso abusivo e a dependência como problema de saúde ${ }^{(1)}$. Suas consequências não se limitam a questões de segurança, mas, também, estão ligadas intrinsecamente a questões sociais, psíquicas e de saúde, tanto dos usuários quanto das pessoas que estão em seu entorno.

A fissura, caracterizada pelo intenso desejo de consumir determinada substância, é considerada a principal dificuldade enfrentada, já que pode afetar o humor, o comportamento e a cognição(2). Muitas vezes a fissura é apontada como necessidade imprescindível para o corpo, sendo responsável pelo fenômeno conhecido como binge: padrão de consumo intenso, contínuo e repetitivo de crack, que pode durar dias, até que se termine a disponibilidade da substância ou haja a exaustão do usuário(1).

Em estudos, aponta-se que a fissura por crack tem papel fundamental na manutenção do uso, na dificuldade de diminuição ou abandono da substância e nas recaídas ${ }^{(1,3)}$. Por isso, faz-se necessário e primordial estudar a fissura e, com isso, buscar melhores compreensões a respeito dos possíveis fatores relacionados ao maior ou menor nível.

Há de se levar em conta que a utilização de agentes farmacoterapêuticos, como candidatos ao tratamento da fissura, vem apresentando resultados que, em geral, foram classificados como decepcionantes ${ }^{(4)}$. 
Compreendendo-se que os usuários de Substâncias Psicoativas (SPA) são singulares e questões além do próprio efeito da substância interferem no modo como ela é utilizada, neste estudo, teve-se como objetivo a realização de revisão de literatura sistematizada, a fim de identificar publicações relacionadas à influência dos fatores associados à variação dos níveis de fissura, em usuários de crack.

\section{Metodologia}

Objetivando-se buscar produção científica acerca do tema para aprofundamento no assunto, foi realizada revisão sistemática sobre fissura em usuários de crack, expandindo os achados para a seguinte questão norteadora: quais são os fatores que influenciam a variação do nível de fissura em usuários de crack?

A revisão de literatura ocorreu entre os meses de setembro e outubro de 2015 e foram rastreados estudos publicados sobre a temática no Brasil e no exterior.

As bases de dados utilizadas para busca dos estudos foram Publisher Medline (PubMed), Literatura Latino-Americana e do Caribe em Ciências da Saúde (LILACS) e Scientific Electronic Library Online (SciELO). Para busca na LILACS e na SciELO utilizou-se o banco de descritores do Descritores em Ciências da Saúde (DeCs), e para a busca na base de dados PubMed, os termos segundo o Medical Subject Headings (MeSH). Os descritores exatos utilizados para a busca foram: crack cocaine e craving, e seus termos similares, e o descritor não exato CCQ-Brief. Não foram utilizados filtros para a delimitação de ano de publicação e, tampouco, o idioma das publicações.

Os critérios de inclusão foram: estudos quantitativos nos quais se trabalhou a fissura em usuários de cocaína-crack. Todos os artigos rastreados foram avaliados primeiramente por seus títulos e, posteriormente, resumos. Como critérios de exclusão, não foram aceitos estudos de caso, clínicos, qualitativos e revisões de literatura em que o foco fosse a medicação, bem como em que fossem utilizadas metodologias pouco claras ou fora da temática. Todos os artigos que atenderam o critério de inclusão, ou não, apresentavam elementos suficientes para determinar sua exclusão foram lidos na íntegra.

Para extração dos dados nos artigos, criou-se instrumento contendo as seguintes informações: autores, ano de publicação, local do estudo, periódico de publicação, tamanho da amostra e fatores associados à fissura de crack.

É importante destacar, aqui, o percurso para a obtenção dos artigos na íntegra, visto que a maioria se apresentava em caráter privado. $\mathrm{O}$ primeiro passo adotado, em face da indisponibilidade do artigo na íntegra nas bases de dados, foi a pesquisa na página da web Research Gate, a qual é caracterizada como rede social onde diversos autores disponibilizam seus estudos. Após isso, os dois artigos faltantes foram obtidos por meio de correspondência eletrônica com os autores. Acredita-se na necessidade de divulgação desses passos, visto que muitos autores optam por realizar revisões apenas com artigos de livre acesso, podendo, assim, prejudicar a qualidade de seus estudos.

\section{Resultados}

Como resultado, neste estudo, foram rastreados por meio das buscas 101 estudos, no total, sendo 57 da PubMed, 20 da SciELO e 24 da LILACS. O primeiro passo para a seleção dos artigos consistiu na leitura dos títulos e resumos. Durante esse procedimento, 69 estudos foram descartados, sendo 18 estudos qualitativos, cinco estudos de caso, 33 estudos clínicos com foco em medicação, seis estudos de revisão da literatura e sete estudos fora da temática. Outros 12 estudos foram descartados por serem duplicados, restando, assim, 20 textos para serem lidos na íntegra. Dos estudos lidos na íntegra, 11 foram descartados por terem foco em medicação, restando, assim, nove artigos a serem considerados para esta revisão. Na Figura 1 é apresentado o percurso para seleção dos artigos.

Na Figura 2 é apresentado o instrumento utilizado para a coleta de dados dos artigos selecionados.

Entre os estudos selecionados, quatro foram redigidos em língua portuguesa e cinco em língua inglesa. Em relação à metodologia utilizada para o desenvolvimento dos estudos, em seis foi utilizado corte transversal, em dois, longitudinal e, em um, tradução e adaptação de escalas para a língua portuguesa. Os artigos apresentaram o número de participantes distintos, variando de 20 a 205 entrevistados, contudo, a maioria (seis) apresentou amostra de até 66 entrevistados.

Em relação às escalas utilizadas, observou-se que em apenas dois estudos não foram utilizadas escalas validadas $^{(10-11)}$, nos outros setes artigos analisados foram utilizadas 13 escalas distintas. As escalas que mais se repetiram foram: Addiction Severity Index-6, encontrada em quatro estudos ${ }^{(6-8,13)}$, e escala Cocaine Craving Questionnaire-Brief, encontrada em três estudos $^{(5-6,9)}$.

Observando-se os instrumentos utilizados pelos autores para a coleta de dados, com exceção de um estudo(6), nos demais (oito) coletaram-se questões referentes ao perfil sociodemográfico dos entrevistados. 


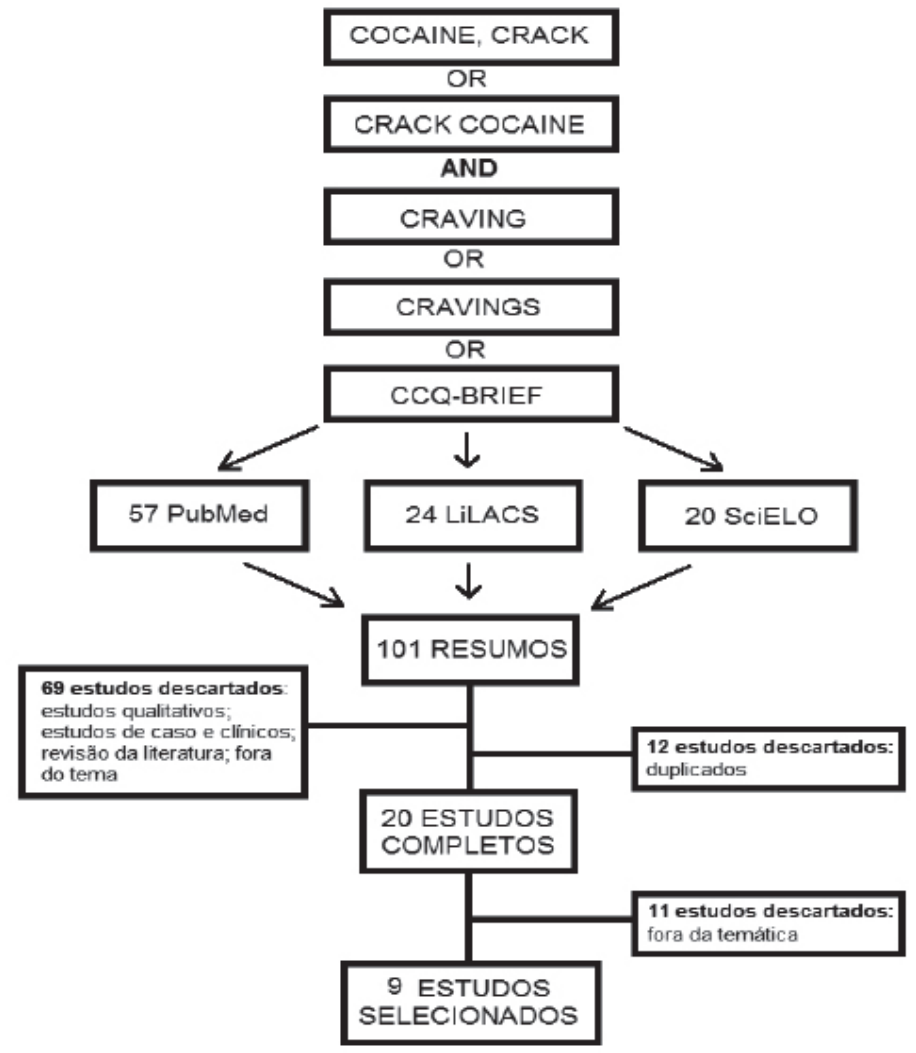

Figura 1 - Percurso da revisão de literatura sistematizada. Pelotas, RS, Brasil, 2015

\begin{tabular}{|c|c|c|c|c|}
\hline Primeiro autor & Local/ano & Periódico & Amostra & Fatores associados \\
\hline Lappann $^{(5)}$ & Brasil/2015 & R. Eletr. Saúde Mental Álcool e Drogas & $n=20$ & Tempo de uso do crack \\
\hline Kluwe-Schiavon(6) & Brasil/2015 & J. Brasileiro de Psiquiatria & $n=125$ & $\begin{array}{l}\text { Hábitos de vida não } \\
\text { saudáveis e ansiedade }\end{array}$ \\
\hline Ismael(7) $^{(7)}$ & Brasil/2014 & R. Brasileira de Psiquiatria & $n=66$ & $\begin{array}{l}\text { Padrão de uso do crack e } \\
\text { ansiedade }\end{array}$ \\
\hline Viola $^{(8)}$ & Brasil/2013 & Estudos de Psicologia & $n=120$ & Relações familiares \\
\hline Viola $^{(9)}$ & Brasil/2012 & Drug and Alcohol Dependence & $n=45$ & Atividade profissional \\
\hline Silveira(10) & Brasil/2006 & Addictive Behaviors & $n=205$ & Níveis de fissura \\
\hline Magura(11) $^{(11}$ & EUA/2000 & Addictive Behaviors & $n=66$ & Utilização de álcool \\
\hline Labigalini Junior $^{(12)}$ & Brasil/1999 & J. of Psychoactive Drugs & $n=25$ & Utilização de Cannabis \\
\hline $\operatorname{Reid}^{(13)}$ & EUA/1998 & Drug and Alcohol Dependence & $n=20$ & Utilização de nicotina \\
\hline
\end{tabular}

Figura 2 - Características dos artigos rastreados pela revisão de literatura $(n=9)$. Pelotas, RS, Brasil, 2015

O perfil dos entrevistados variou entre os estudos, em dois artigos foiapresentada amostra exclusivamente do sexo feminino(6,8), em outros dois artigos, amostra exclusivamente do sexo masculino ${ }^{(7,12)}$, e um estudo com entrevistados convencionalmente selecionados, para que metade fosse do sexo masculino e metade do sexo feminino ${ }^{(9)}$, os quatro demais estudos, nos quais a seleção da amostra foi aleatória, foram compostos de entrevistados predominantemente do sexo masculino, com prevalência variando de 56 a $90 \%$.

Os entrevistados foram, majoritariamente, jovens e solteiros. Entre os estudos em que se descreveu o nível de escolaridade dos entrevistados, apenas em um observou-se que os usuários apresentavam média de 12 anos ou mais de estudo(7), nos demais encontrou-se baixa escolaridade ${ }^{(5,9,12)}$.

$\mathrm{Em}$ apenas dois artigos foi apresentado o detalhamento dos níveis de fissura. No primeiro estudo foi apresentada a média de craving dos entrevistados, segundo a escala CCQ-Brief, em que o resultado aponta para nível grave $(\geq 23 \text { pontos })^{(5)}$. E, no segundo estudo, $29 \%$ dos entrevistados referiram ter sentido craving intenso, $39,5 \%$ relataram o craving moderado e $31,5 \%$ relataram craving leve ${ }^{(10)}$.

\section{Discussão}

Em todos os artigos analisados realizaram-se estudos das variáveis que poderiam influenciar a 
fissura pelo crack dos entrevistados. Observou-se que o aumento dos níveis de fissura está associado positivamente ao tempo de uso de crack, isto é, quanto maior o tempo de uso da substância, maiores serão os níveis de fissura(5). Os autores ressaltam, ainda, que a quantidade utilizada não interfere na alteração desses níveis. Contudo, em outro estudo, o padrão de uso do crack mostrou-se como fator associado ao aumento dos níveis de fissura(7).

Outro ponto analisado em alguns estudos foi a existência de interferência nos níveis de fissura e a realização de alguma atividade profissional. Foi constatado que os níveis de fissura eram menores entre as pessoas que exerciam atividades profissionais e pessoas com níveis de fissura elevados não conseguiam executar tais funções ${ }^{(8-9)}$.

Os prejuízos que usuários de crack apresentam na tomada de decisão, aliados à dificuldade em lidarem com o craving, são fatores que podem influenciar o ciclo de recaídas, demissão do emprego, prejuízos financeiros e até desagregação familiar ${ }^{(9)}$. Cabe destacar que, neste estudo, a amostra selecionada se apresenta como poliusuária, ou seja, pessoas que fazem uso de diversas substâncias psicoativas, além do crack, que afetam o sistema cognitivo, o que poderia interferir no resultado derivado desse.

Também levantou-se o fato de que as relações familiares interferem na fissura, visto que pessoas com histórico de negligência na infância apresentaram maiores níveis de fissura em relação àqueles que possuíam esse histórico ${ }^{(8)}$. Apesar de, neste estudo, a negligência na infância ser apresentada como possível fator que poderia influenciar os níveis de fissura do crack, o fato de ter sido realizado apenas com mulheres é limitador, não podendo os resultados deste serem aplicados na população do sexo masculino.

Ao analisar os resultados presentes em outro estudo, identificou-se que a realização de hábitos de vida não saudáveis também foi apontada como possível fator que interfere nos níveis de fissura. A falta de alimentação, a não ingestão de água e a ausência ou diminuição de períodos de sono foram fatores que influenciaram o aumento da fissura ${ }^{(6)}$. Assim, sem o intuito de apresentar normas prescritivas, ao analisar os resultados neste estudo, destaca-se a necessidade de o indivíduo estabelecer certos padrões ou hábitos mais saudáveis, os quais influenciam positivamente o metabolismo do organismo, bem como a diminuição dos sintomas de abstinência e, consequentemente, os níveis de fissura que podem se evidenciar. Como ponto negativo neste estudo, destaca-se que o mesmo foi realizado apenas com população do sexo feminino, limitando a extrapolação dos seus resultados para outras populações.

A interferência de outras SPA nos níveis de fissura também foi estudada. No estudo em que foi analisada a interferência do álcool na fissura dos entrevistados, nota-se que os indivíduos que têm dificuldade em lidar com o consumo de cocaína-crack são mais propensos ao uso do álcool como mecanismo para a diminuição da fissura(11). E, no estudo em que foi realizada análise da interferência da maconha como forma de tratamento para a diminuição do uso do crack e dos níveis de fissura, obteve-se o resultado de que $68 \%$ dos entrevistados deixaram de utilizar o crack, e que o uso da Cannabis reduziu a fissura e produziu mudanças subjetivas e concretas em seus comportamentos ${ }^{(12)}$. Os resultados nesses estudos apresentaram a utilização de outras substâncias psicoativas como estratégia para amenizar os sintomas da abstinência e os níveis de fissura do crack. No entanto, há de se considerar a necessidade de avaliação mais profunda do uso em questão, a fim de evitar o abuso dessas outras substâncias que, assim como o uso abusivo de crack, pode levar a prejuízos, tanto no âmbito físico e psíquico quanto no social.

Já nas análises da interferência, provocada pela utilização do tabaco, demonstrou-se, nos resultados, que a nicotina aumenta os níveis de fissura(13). Portanto, pode-se notar que a utilização do álcool e/ ou da maconha podem servir como mecanismo de proteção para a diminuição dos níveis de fissura, porém, a utilização do tabaco pode acarretar o aumento desses níveis.

Finalizando, os fatores associados aos níveis de fissura, a presença dos sintomas de ansiedade foi apresentada por diversos autores como fator que interfere diretamente no aparecimento e aumento desses níveis ${ }^{(6-7,12-13)}$. A conclusão de que a ansiedade se apresenta como questão que influencia positivamente os níveis de fissura faz dela importante fator, que deve ser identificado e analisado como alvo principal, em estudos futuros. A identificação de suas causas eleva o cuidado em saúde baseado apenas nos aspectos biológicos para outro patamar, a partir do cuidado biopsicossocial.

\section{Conclusão}

Nos artigos, estudou-se a influência das seguintes variáveis sob o desfecho de níveis de fissura em usuários de crack: tempo de uso do crack; padrão de uso do crack; ocupação profissional; relações familiares; estratégias de redução de 
danos; utilização concomitante de álcool, maconha e tabaco e sintomas de ansiedade. Contudo, ressalta-se que em nenhum desses estudos foram analisados todos esses fatores concomitantemente, evidenciando, assim, lacuna investigativa em virtude da necessidade de testar a influência dessas variáveis, ajustadas entre si, sob o desfecho de níveis de fissura em usuários de crack.

Considerando que a fissura pode acarretar consumo intenso de substâncias psicoativas e, em virtude disso, pode ocasionar diversos problemas para os usuários, de ordem social ou de saúde, acredita-se que novos estudos quantitativos nos quais se investigue a interferência de fatores na fissura, com amostra representativa, devem ser realizados para que ocorra compreensão da melhor forma de ação, com vistas a ajudar os usuários no enfrentamento do problema.

\section{Referências}

1. Chaves TV, Sanchez ZM, Ribeiro LA, Nappo SA. Fissura por crack: comportamentos e estratégias de controle de usuários e ex-usuários. Rev Saúde Pública. 2011; 45(6):1168-75.

2. Coelho LRM, Sá LGC, Oliveira MS. Estratégias e Habilidades de Enfrentamento de Usuários de Crack em Tratamento. Rev Psicol IMED. 2015; 7(2):99-109.

3. Picoli MMC. Adesão de Usuários de Crack ao Tratamento num Caps I do Interior do Rio Grande do Sul [dissertação]. Florianópolis (SC): Universidade Federal de Santa Catarina; 2013.

4. Fischer B, Kuganesan S, Gallassi A, Malcher-Lopes $\mathrm{R}$, Brink W, Wood E. Addressing the stimulant treatment gap: A call to investigate the therapeutic benefits potential of cannabinoids for crack-cocaine use. Int J Drug Policy. 2015;26:1177-82.

5. Lappann NC, Machado JSA, Tameirão FV, Benjamim MLN. Craving pelo crack nos usuários em tratamento no centro de atenção psicossocial. SMAD, Rev. Eletrônica Saúde Mental Álcool Drog. 2015; 11(1):19-24.

6. Kluwe-Schiavon B, Tractenberg SG, Sanvicente-Vieira B, Rosa CSO, Arteche AX, Pezzi JC, et al. Propriedades psicométricas da Cocaine Selective Severity Assessment (CSSA) em mulheres usuárias de crack. J Bras Psiquiatria. 2015;64(2):115-21.

7. Ismael F, Baltieri DA. Role of personality traits in cocaine craving throughout an outpatient psychosocial treatment program. Rev Bras Psiquiatria. 2014;36(1):24-31.

8. Viola TW, Tractenberg SG, Pezzi JC, Kristensen $\mathrm{CH}$, Grassi-Oliveira R. Childhood physical neglect associated with executive functions impairments in crack cocaine- dependent women. Drug Alcohol Dependence. 2013; 132:271-76.

9. Viola TW, Cardoso CO, Francke ID, Gonçalves HA, Pezzi JC, Araújo RB, et al. Tomada de decisão em dependentes de crack: um estudo com o lowa Gambling Task. Estudos Psicol. 2012;17(1):99-106.

10. Silveira DX, Doering-Silveira E, Niel M, Jorge MR. Predicting craving among cocaine users. Addictive Behaviors. 2006;31:2292-7.

11. Magura S, Rosenblum A. Modulating effect of alcohol use on cocaine use. Addictive Behav. 2000;25(1):117-22. 12. Labigalini Junior E, Rodrigues LR, Silveira DX. Therapeutic use of cannabis by crack addicts in Brazil. J Psychoactive Drugs. 1999;31(4):451-5.

13. Reid MS, Mickalian JD, Delucchi KL, Hall SM, Berger SP. An acute dose of nicotine enhances cueinduced cocaine craving. Drug and Alcohol Dependence. 1998;49:95-104. 